\title{
Enabling personalized cancer medicine decisions: The challenging pharmacological approach of PBPK models for nanomedicine and pharmacogenomics (Review)
}

\author{
IOANNIS S. VIZIRIANAKIS ${ }^{1,2}$, GEORGE A. MYSTRIDIS ${ }^{1}$, KONSTANTINOS AVGOUSTAKIS ${ }^{3}$, \\ DIMITRIOS G. FATOUROS ${ }^{4}$ and MARIOS SPANAKIS ${ }^{5}$
}

\begin{abstract}
${ }^{1}$ Laboratory of Pharmacology, Department of Pharmaceutical Sciences, School of Health Sciences, Aristotle University of Thessaloniki, Thessaloniki GR-54124, Greece; ${ }^{2}$ Department of Life and Health Sciences, University of Nicosia, 1700 Nicosia, Cyprus; ${ }^{3}$ Laboratory of Pharmaceutical Technology, Department of Pharmaceutical Sciences, University of Patras, Patras GR-26504; ${ }^{4}$ Laboratory of Pharmaceutical Technology, Department of Pharmaceutical Sciences, Aristotle University of Thessaloniki, Thessaloniki GR-54124; ${ }^{5}$ Computational BioMedicine Laboratory, Institute of Computer Science, Foundation for Research and Technology-Hellas, Heraklion GR-71110, Crete, Greece
\end{abstract}

Received September 25, 2015; Accepted October 27, 2015

DOI: $10.3892 / o r .2016 .4575$

\begin{abstract}
The existing tumor heterogeneity and the complexity of cancer cell biology critically demand powerful translational tools with which to support interdisciplinary efforts aiming to advance personalized cancer medicine decisions in drug development and clinical practice. The development of physiologically based pharmacokinetic (PBPK) models to predict the effects of drugs in the body facilitates the clinical translation of genomic knowledge and the implementation of in vivo pharmacology experience with pharmacogenomics. Such a direction unequivocally empowers our capacity to also make personalized drug dosage scheme decisions for drugs, including molecularly targeted agents and innovative nanoformulations, i.e. in establishing pharmacotyping in prescription. In this way, the applicability of PBPK models to guide individualized cancer therapeutic decisions of broad clinical utility in nanomedicine in real-time and in a cost-affordable manner will be discussed. The latter will be presented by emphasizing the need for combined efforts within the scientific borderlines of genomics with nanotechnology to ensure major benefits and productivity for nanomedicine and personalized medicine interventions.
\end{abstract}

Correspondence to: Professor Ioannis S. Vizirianakis, Laboratory of Pharmacology, Department of Pharmaceutical Sciences, School of Health Sciences, Aristotle University of Thessaloniki, Thessaloniki GR-54124, Greece

E-mail: ivizir@pharm.auth.gr

Key words: personalized medicine, pharmacokinetics, pharmacodynamics, pharmacotyping, PBPK models, nanomedicine, drug delivery

\section{Contents}

1. Introduction

2. The economic burden and cost-effectiveness of PGx to enable personalized cancer medicine decisions

3. Development of PBPK models and advanced ex vivo pharmacological assays to support PTx and implement clinical pharmacology guidelines

4. Model-based approaches for enabling the development of cancer-targeted delivery of nanomedicines by simulating tumor microenvironment heterogeneity

5. Moving toward the implementation of PBPK models in nanomedicines: The case of tumor modeling and cancer therapy

6. Future perspectives in advancing PBPK models to ensure practical clinical utility of cancer therapeutic interventions and PTx

\section{Introduction}

Currently, the development of powerful and smart highthroughput tools in translational medicine along with nanotechnological applications enabling information-based medicine have revived the hope for the broader application of pharmacogenomics (PGx) for most, if not all, individual patients (see Table I for term definition) (1-10). By extending such a notion from PGx to routine healthcare and drug prescription ( $\mathrm{Rx})$, it means that this transition is better served by achieving pharmacotyping (PTx) in drug delivery $(6,8,9)$. Further support is gained through expanding the clinical application of innovative approaches and technicalities happening in the era of: i) genomic profiling; ii) biomedical imaging; iii) synthetic, biology-based, cell engineering to advance drug delivery entities; iv) population-based modeling as predictive tools of pharmacokinetic (PK) parameters of drugs (absorption, distribution, metabolism and excretion; ADME) and v) innovative approaches and tools on patient electronic data 
Table I. Definition of terms.

CYP genetic variation refers to various genetic polymorphisms existing in cytochrome P450 (CYP) drug metabolizing enzymes that contribute to altered PK behavior due to dysfunction of drug metabolism and thus to limited drug therapy outcomes.

Personalized medicine joins the gap between the clinical setting and various health-related basic disciplines with genomics in a way to increase precision of prognosis, diagnosis, and therapy outcomes of individuals in an affordable way. The latter implies in real-time the stratification of patients suffering the same complex illness (e.g., cancer, cardiovascular disorders), thus referring to the clinical translation of genomic knowledge into practical clinical utility for disease risk assessment, diagnostic profiles, therapeutic delivery and new drug development approaches.

Pharmacogenomics (PGx) bridges the gap between pharmacology and genomics toward unveiling the pharmacological response heterogeneity, providing the promise of the clinical implementation of molecular knowledge into the pharmacokinetic/ pharmacodynamic (PK/PD) level as well as moving drug prescription to pharmacotyping (e.g. the individualized drug selection dosage schemes based on specific patient clinical characteristics and genetic make-up).

Pharmacotyping (PTx) applies to drug prescription (Rx) and describes the process where the physician's selection of specific drugs and their dosage schemes is also based on each individual patient's genetic make-up.

management, clinical support and routine healthcare. These scientific breakthroughs empower the speed and the productivity in developing innovative molecularly targeted drugs and nanotheranostics with improved clinical safety and efficacy profiles (11-20). The latter coincides with the movement from an Rx process mainly based on the physician's own experience into a more highly digitized and integrated workflow platform to aid the administration of individualized drug dosage schemes for personalized medicine.

As a matter of fact, the interdisciplinary infrastructure and methodologies needed to broadly enable PTx in the clinical setting is depicted in Fig. 1 (for a more extensive overview see Ref. 7). In particular, such a diagrammatic presentation exemplifies the environment to maximize outcomes and achieve improved efficacy and safety profiles of clinical practical utility for personalized medicine decisions. That means that the proper translational capacity for all the involved disciplines and technologies has to be clinically validated in terms of precise clinical diagnosis and drug prescription dosage scheme selection by healthcare practitioners. The latter refers to advancements such as: i) the 'omics'-related for molecular diagnostics and PGx; ii) the computerized/information-based platforms in data and text mining for bioinformatics and biostatistics; and iii) the PK/pharmacodynamic (PK/PD) guidelines for pharmacovigilance, molecular and clinical pharmacology. By enabling this direction, the establishment of PTx in drug prescription is served. Last, but not least, the integration of nanotechnology, imaging technologies and advanced cell therapies impacting nanomedicine applications are also better facilitated and implemented in the clinical setting, as shown in Fig. 1.

Following such a roadmap in Rx it must also be considered that the inter-individual response heterogeneity (patient phenotype) noted in clinical pharmacology and the incidence of adverse drug reactions (ADRs) are related to various factors and parameters affecting the risk/benefit therapeutic ratio that refer to: i) the molecular pathophysiology of diseases as well as their severity; ii) the co-administration of medicines that might result in drug interactions; iii) any existed co-morbidities; iv) the functional status of vital organs, i.e. liver and kidneys contributing to PK behavior of drugs; v) the age of the individual patient; vi) the person's lifestyle, i.e. smoking, caffeine intake; vii) the patient's compliance (adherence) to the physician's guidance to prescribed therapy; and also viii) the personal genetic make-up, or alternatively, the existing human genome variability in genes involved in $\mathrm{PK} / \mathrm{PD}$ processes ensuring drug effects in the body; i.e. the genetic polymorphism of various gene alleles referring to drug metabolizing enzymes, transporters and receptors (21-32). Simultaneously, the successful address of such PK/PD issues represents the gold-standard target upon developing drug nanodelivery vehicles.

\section{The economic burden and cost-effectiveness of PGx to enable personalized cancer medicine decisions}

It has been estimated that drug response heterogeneity for marketed drugs significantly contributes to the increase of healthcare costs, the rate of in-patient hospitalization and mortality index (33-37). Although there is a widespread interest in personalized medicine, the broad application of PGx testing implies that the validation of the clinical improvement outcome must be clearly demonstrated in a cost-effective manner. Undoubtedly, however, only a limited number of studies have addressed cost-effectiveness issues of PGx applications in the clinical setting (38-41). To this end, recent studies assessing the pharmacoeconomic benefits of thiopurine methyltransferase (TPMT) PGx testing have shown a favorable cost-effectiveness ratio, by analyzing either the outcome achieved in children suffering from acute lymphoblastic leukemia (ALL), or relevant pharmacovigilance data referring to the emergence of $\operatorname{ADRs}(39,41)$. On the contrary, the lack of standardized PGx economic models has undoubtedly emerged in the case of CYP genetic variation, by trying to evaluate the economic burden vs. the clinical benefits of PGx testing upon prescribing antipsychotics in routine healthcare (38). In addition, the complexity in undertaking a trial-based evaluation of the cost-effectiveness of PGx tests such as the application of TPMT for prescribing azathioprine in patients suffering autoimmune diseases has recently been shown (40). Moreover, 


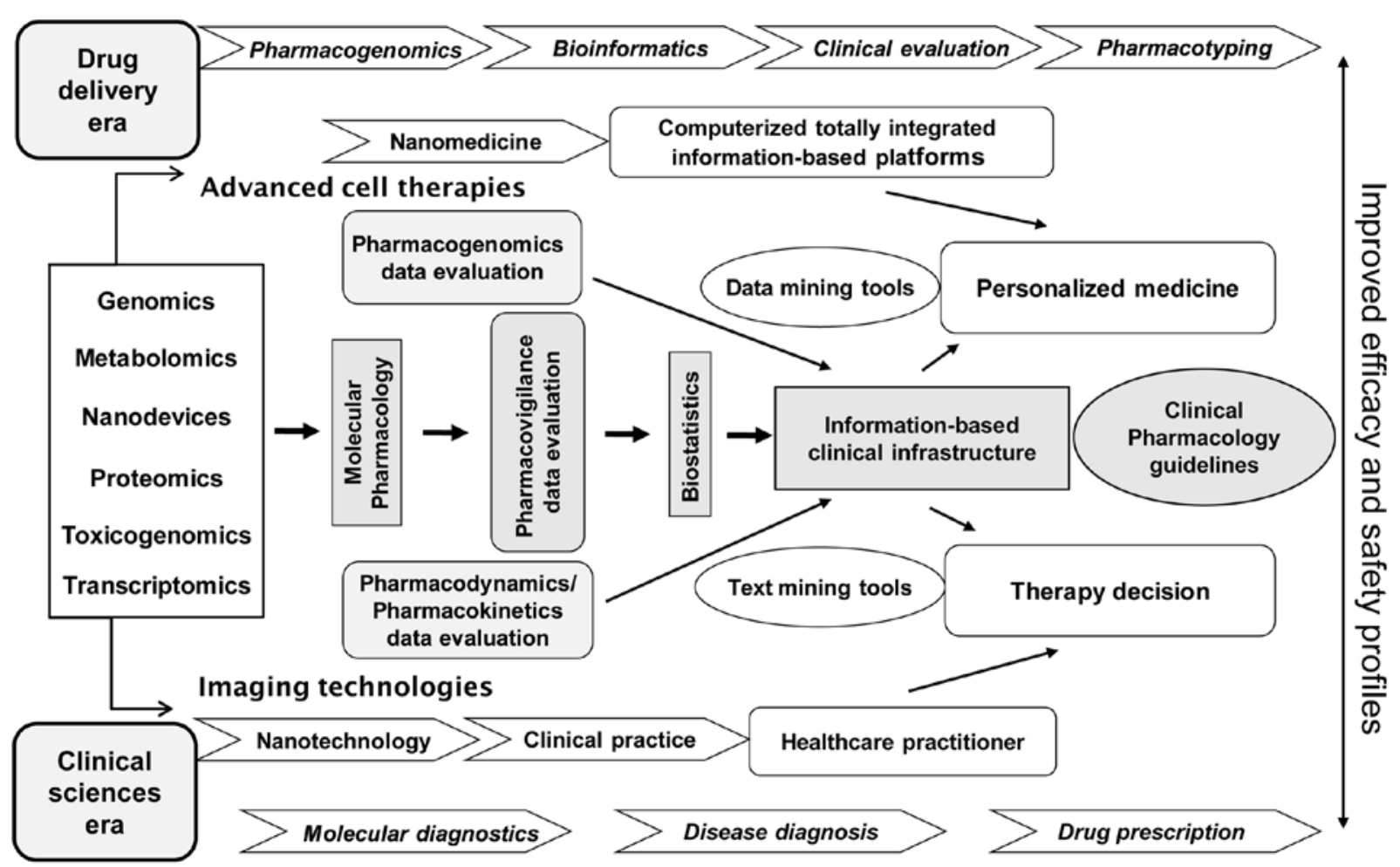

Figure 1. Depiction of scientific efforts and methodologies contributing to personalized medicine decisions toward advancing broader drug prescription profiles (see text for details).

the economic evaluation of PGx testing is now considered a main barrier hindering the implementation of clinical practice with PGx knowledge. Importantly, before moving toward the routine application of PGx concepts in the healthcare system and establishing PTx, the clear demonstration of the economic benefits gained must be addressed to accompany the already assured clinical benefits of any PGx testing. Such a direction will allow cost-effectiveness analysis to document the relative cost/benefit ratios of PGx interventions compared to current clinical practices and create the framework for healthcare providers to make reimbursement decisions. Importantly, the limited evidence accumulated thus far in analyzing the economic value of personalized medicine tests also restricts any proper informed decision-making and assessment of genomic priorities (42). Complementary, in order to advance practical clinical utility of personalized medicine decisions, the cost-effectiveness in addition to the clinical effectiveness of PGx tests for decision makers should simultaneously be undertaken in a robust and timely manner (43).

3. Development of PBPK models and advanced ex vivo pharmacological assays to support PTx and implement clinical pharmacology guidelines

The PK and PD behavior of drugs is known to be affected by either drug interactions or genetic polymorphism of genes involved in drug actions, thus leading to altered plasma therapeutic concentrations (inefficiency or toxicity) and also to modulated receptor affinity (sensitivity) $(8,9)$. The future application of physiologically based pharmacokinetic (PBPK) models to shape the practical utility of personalized/stratified medicine and ensure PTx is shown in Fig. 2.Indeed, the accuracy and predictability potential of the applied methodologies and model approaches for assessing the drug plasma concentrationtime profile and clearance in humans has been recently verified $(44,45)$. This, however, implies the development of algorithms to improve the statistical power and robustness upon mathematical integration and PBPK modeling of drug effects by facilitating the clinical exploitation and inference of genome-wide association studies and PGx tests (46-48). In contrast, PGx knowledge for drugs is now considered an integral part of therapeutics and drug development, since a number of predictive PGx biomarkers to assess the safety and clinical efficacy profiles of individual marketed drugs have been validated by drug regulatory agencies (e.g. the FDA and EMA) $(32,49)$. To this end, the development of PBPK models, implemented together with system pharmacology approaches (assessing predictive PGx biomarkers), represents a promising platform where in real-time the assessment of both patient- and drug-related factors can be inter-correlated for individual patient populations. Alternatively, the latter means the elaboration of a multidisciplinary environment in order to assess both drug interactions and PGx data to be effectively incorporated to guide Rx, thus achieving PTx.

The need to empower the predictive capacity of ex vivol in silico pharmacological assays, particularly for complex and multifactorial diseases (e.g. cancer) to advance translational medicine capacity, still represents an unmet need upon new drug development efforts. The demand for obtaining improved productivity outcomes in the preclinical phase is further stressed by analyzing data from specialized sources referring to registered medicines under development and in 


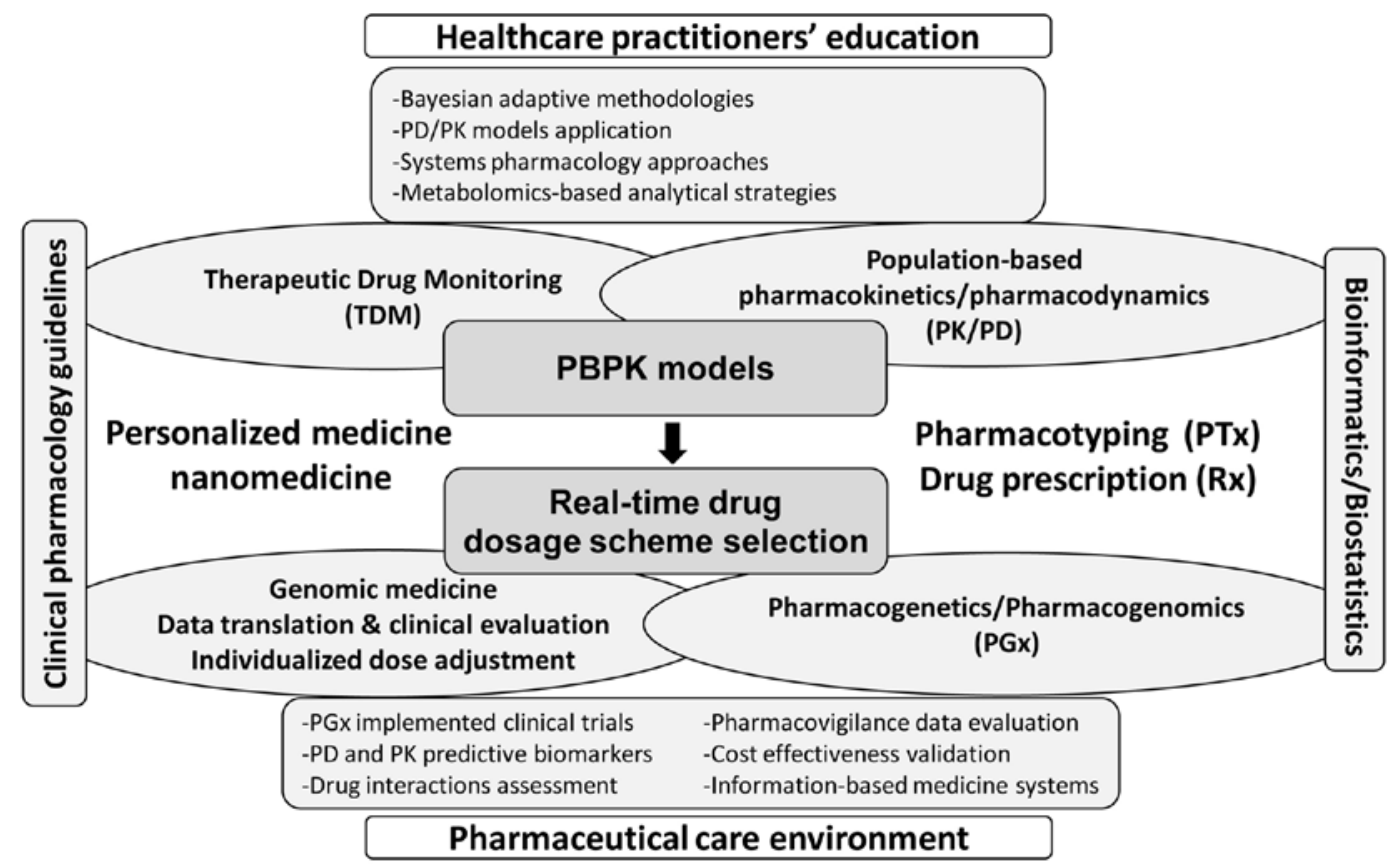

Figure 2. A scheme of pharmacotyping in the drug prescription process is depicted within the context of personalized medicine and nanomedicine. Specific research and/or clinically-oriented methodologies are presented with focus on PBPK modeling to allow real-time drug dosage-scheme selection toward achieving personalized medicine broader utility decisions in drug delivery and therapeutics (see text for details).

clinical trials $(50,51)$. However for example, by considering the era of innovative anticancer therapeutics, a major question then is raised amenable to further analysis pending on how to better succeed in this task? At first, the development of predictive biomarkers applied as companion diagnostics of molecularly targeted anticancer drugs to achieve personalized treatment currently represents an established practice toward assessing tumor genetic make-up variability. Secondly and in contrast, it is a well-established notion in clinical practice that pharmacological response variability is also attributed to various drug-, patient- and/or environmental-related factors (8). The former has been successful to date in clinical practice through mainly in vivo pharmacology advancements and the latter through PK developments. By taking both such notions into consideration, it is clear that in achieving a broadly successful personalized cancer therapy option, both tumor cell and genome variability as well as individualized drug dosage scheme delivery (e.g. PTx) have to be inter-correlated and simultaneously applied in routine clinical practice $(8,9,32)$. In addition, importantly, the potential to accelerate such innovative anticancer therapeutic developments, in a way to match the personalized cancer therapy needs and expectations in clinical practice, will require specific collaborative attempts between academia, industry, and regulatory agencies $(19,52)$. In other words, the previously gained experience from both in vivo pharmacology experimentation and therapeutic drug monitoring methodology both in research and clinical practice have to be implemented with suitably designed, validated and developed molecular diagnostics to elucidate the missing links in knowledge for personalized medicine decisions. To this end, pharmacologists must positively work to advance broader practical utility decisions by directing and guiding the establishment of a research-based, regulatory-oriented and clinically-focused personalized medicine environment for various pharmacological classes of therapeutics and illnesses $(8,9,32)$. To this end, if the successful development of quantitative PGx models for translation medicine is achieved, than the genetically-guided drug dose adjustment can be best utilized to improve drug development and routine clinical practice outcomes (52-54). In fulfilling this task of practical clinical utility, the inter-convergence of genomic with clinical information can occur and bring together clinicians and scientists of basic sciences, thus accelerating the pace of PTx in $\mathrm{Rx}$.

By taking into consideration the deficit in scientific knowledge, the approach to accelerate the development of more powerful innovative anticancer therapeutics is by discovering methods by which to improve early clinical anticancer drug evaluation through structured and rational trial designs that incorporate predictive PK, PD, PGx and intermediate end-point biomarkers (51). In addition, it is also crucial to discover ways to enrich methodologies facilitating the integration of PGx into know-how strategy of the mainstay drug development pipeline (55). The latter implies that the suitable organization and application of population-based PK/PD ex vivo pharmacology modeling is also vital to support the integration of genomic knowledge in routine clinical practice and $\mathrm{Rx}$ by hastening the movement toward personalized medicine (56). Moreover, it is evident from the previously gained in vivo pharmacology experience in drug development that the efficient clinical translation of genomic knowledge coincides with successfully addressing the issues related to the adjustment of clinical pharmacology guidelines toward personalized medicine concepts. Alternatively, the implementation of clinical pharmacology guidelines with PGx knowledge through the development of advanced PBPK models represents one of the most crucial 
Table II. Application of PBPK models in selected nanomedicine studies.

\begin{tabular}{ll}
\hline Nanoformulation & \multicolumn{1}{c}{ PBPK model application } \\
\hline Silver nanoparticles & Mouse whole-body PBPK model \\
PLGA nanoparticles & Mouse semi-mechanistic PBPK model \\
Nano-TiO 2 particles & Human whole-body PBPK model \\
Aluminum nanoparticles & In vitro-in vivo extrapolation (IVIVE) PBPK model \\
& in a population of rat alveolar macrophages \\
Quantum dots & Mouse whole-body PBPK model \\
99mTc-labeled nanoparticles & Human whole-body PBPK model \\
Silver nanoparticles & Rat whole-body PBPK model \\
Gold-dendrimer composite nanodevices & Mouse whole-body PBPK model \\
Nanocarrier-mediated delivery of various drugs & Semi-mechanistic PBPK model of \\
hepatocellular carcinoma (HCC) tumor cells \\
PEG-coated PAA nanoparticles & Rat whole-body PBPK model \\
Metal nanoparticles & Rat-whole body PBPK model \\
Gold nanoparticles & In vitro and in silico methods for assessing the \\
& pulmonary translocation and biodistribution \\
& of NPs towards replacement of in vivo studies \\
PEG-Gold nanoparticles & Mouse membrane-limited whole-body PBPK model \\
Silver nanoparticles of different sizes & Rat-PBPK model of 2 and 5 compartments
\end{tabular}

Silver nanoparticles

PLGA nanoparticles

Nano- $\mathrm{TiO}_{2}$ particles

Quantum dots

T9m-labeled nanoparticles

Silver nanoparticles

Gold-dendrimer composite nanodevices

PEG-coated PAA nanoparticles

Metal nanoparticles

PBPK, physiologically based pharmacokinetics. elements for enabling the practical utility of personalized medicine and applying PTx concepts for drug delivery worldwide $(8,9)$. This direction also necessitates overcoming barriers hindering efficient clinical translation and implementation of PGx knowledge from bench to bedside. To this end, it is notable that PGx guidelines for dose recommendation schemes of specific pharmacological drug classes have already been initiated and proposed (57-62). To foster the applicability and practical utility of these methodologies through the use of PBPK/PGx models, the assessment of any ethical, social and cost-benefit impacts should also be addressed before the transfer of techniques used in genomic-related research laboratories to diagnostic laboratories $(8,9,32,54)$. Subsequently, the modeling of the tumor microenvironment will be outlined to further stress the usefulness of the application of PBPKs in the era of nanomedicine and personalized medicine.

\section{Model-based approaches for enabling the development of cancer-targeted delivery of nanomedicines by simulating tumor microenvironment heterogeneity}

One of the most challenging issues in pharmacology and therapeutics relates to the capacity of predicting the PK profile of any administered drug to individual patients and thus to estimate the cellular concentrations reached at the site of action referring either to the diseased organ, or tissue in order to be within the therapeutic ratio range. This prediction of the behavior of drugs plays fundamental and crucial roles in the development of stratified and personalized treatment decisions. Indeed, the latter offers opportunities for pharmacologists and clinicians to generate models and test different scenarios of the effects of drugs on the lesion (organ or tissue) by taking into consideration all the variants, including different genomic backgrounds that eventually might contribute to variation in the pharmacological response and clinical phenotype. Particularly for cancer therapy, tumor microenvironment heterogeneity represents a main obstacle hindering the development of innovative anticancer therapeutics and also limiting the clinical outcomes achieved thus far $(19,63,64)$. Particularly for solid tumors, the role of the lesion microenvironment, blood flow, vessel leakage, oxygen supply, size, specific gene expression and growth rate have been found to play an important role in therapeutic outcomes since they are related with drug delivery (i.e. PK) and consequently with pharmacological action (i.e. PD) (65-68). Despite the fact that chemotherapy dosage schemes in oncology always take into consideration various patient characteristics (i.e. body weight), the applied therapeutic protocols and scaling of the dose are based on nomograms and the optimum fitting of the patient to them instead of considering additional tumor characteristics. Although limited attempts to apply PBPK models for nanoformulations have to date been made (see Table II), the dynamics of such a methodology and the way by which specific factors crucial for the PK profile of nanomedicines could be quantified in models have recently been presented $(69,70)$. Alternatively, the recent advancements in translation medicine, nanomedicine and personal genome sequencing permit the implementation of cancer genome testing into the clinical setting, a fact with major opportunities for personalized therapeutic decisions in oncology; i.e. precision cancer medicine to overcome therapy obstacles and ameliorate the negative outcome that oncologists face in their practice (71-74). Moving forward, the genuine application of PBPK modeling in such an innovative cancer therapy era could help to elucidate tumor heterogeneity properties and 
predict drug behavior through simulation of individual patient tumor characteristics and drug properties. A better understanding of the microenvironment complexity, phenotypic diversity and genetic heterogeneity of tumors through PBPK modeling may lead to the development of early approaches in the drug discovery process that will minimize the attrition rate and increase the productivity outcome for novel anticancer therapeutics. An example of personalized cancer therapy is application of tamoxifen for estrogen receptor-positive breast cancer patients $(75,76)$. Tamoxifen is a pro-drug that undergoes extensive hepatic and gut wall metabolism thus forming metabolites with different response effects (77). Moreover, CYP2D6 function and genetic polymorphism has been proven to be the rate-limiting step upon the formation of its main active metabolite 4-hydroxy-N-desmethyltamoxifen (endoxifen). As a consequence, CYP2D6 PGx knowledge allows the prediction of the individual patient pharmacological response and hence the clinical outcome of tamoxifen-treated breast cancer women $(75,77,78)$. In parallel, the clinical impact of drug-drug interactions with concomitant use of selective serotonin reuptake inhibitors such as paroxetine, a strong CYP2D6 inhibitor, has also been described $(79,80)$. Such clinical PGx knowledge has been applied in tamoxifen-based PBPK approaches in order to provide a more precise clinical understanding of any required in vitro-in vivo extrapolation regarding a drug's PK profile evaluation (81). Consequently, tamoxifen-based PBPK modeling provides the opportunity for infrastructure creation for assessing whether potential co-administration of endoxifen with tamoxifen could successfully address genetic variability chemotherapy issues and achieve a sustained pharmacodynamic profile in treated breast cancer patients (82). At the same time, this knowledge appears to stimulate the design of multiscale mechanistic PK/PD model-based approaches for individualizing the therapeutic dosage schemes for tamoxifen and other anticancer drugs, such as temozolamide for glioblastoma, thus introducing a new era for modern PTx in cancer therapeutics $(83,84)$. The latter obviously may also help to apply the predictive capacity of cancer chemotherapy-based PBPK models in nanomedicine development to achieve optimum nanoformulation concentration profiles needed in tumor-targeted cells. In contrast, scientific interdisciplinary efforts in the era of nanomedicine hold promise to seize the opportunity to apply novel modelbased approaches and enhance the capability for designing and analyzing the properties and the pharmacological effects of cancer therapeutic nanoformulations $(82,85)$. Indicatively, there is on-going research on nanoparticles loaded with tamoxifen for the development of nanoformulations with improved PK profiles and tumor-targeted delivery, thus attempting to overcome the PK/PD and PGx issues negatively impacting the clinical outcome, as mentioned above $(86,87)$. Furthermore, in order for the broad exploitation of such innovative approaches, nanoformulations of anticancer drugs such as paclitaxel, docetaxel, cisplatin and doxorubicin are in development and under clinical evaluation (88-91).

In addition to drug-improved profiles and clinical research, recently published model-based PK studies describe the impact of the physicochemical properties of nanoformulations and theranostics on their in vivo kinetic profile as delivery systems and/or therapeutic molecules in tumors $(69,92)$. The combination of the above observations already positively affects the development of novel nanomedicines where their construction, size and physical properties contribute to their targeted delivery to the lesion site. Moreover, it is indicative of the future perspectives presented for the era of nanotechnology and the adoption of all possible data and parameters (model-based, biological, pharmacological and technological) in order to develop nanoformulations with desired PK/PD properties to ensure maximum clinical outcome for most, if not all, patients. To this respect, PBPK modeling represents for complex and multifactorial diseases, such as cancer, an essential and fruitful tool with which to advance future research regarding the disposition of nanoformulations at the site of their action in the body $(70,93,94)$. This needed collaboration from various scientific disciplines of pharmacology, in silico modeling and pharmaceutical nanotechnology shows a tremendous potential for designing sophisticated and clinically effective and safe nanomedicines with either diagnostic and/or therapeutic modality (95-98). Importantly, the usefulness of such nanomedicine-focused PBPK models could directly be exploited for specific cancer (or other disease-related) patient groups such as the pediatric population to help solve issues of pediatric drug development and administration dosage scheme protocols (99). In addition, moving forward, a similar beneficial approach could eventually occur to impact the area of protein cancer therapeutics and relevant formulations, since the peculiar characteristics of this type of drug and their behavior in the body, clearly requires a suitable simulation design and the predictive capacity of more complex PBPK models (i.e. including immune system) to enhance productivity, drug behavior profiles and clinical outcomes (100-102).

\section{Moving toward the implementation of PBPK models in nanomedicines: The case of tumor modeling and cancer therapy}

Undoubtedly, the early applicability of PBPK models for the development of small-molecule drug entities has advanced the predictive capability of their subsequent PK/PD effect profiles which has eventually enhanced the productivity outcomes within the new drug development era (103). Currently, however, PBPK modeling extends toward covering the administration of therapeutic proteins based on the continuously increasing number of biopharmaceuticals reaching the market $(102,104,105)$. From the mechanistic perspective, modeling and simulation of PBPK are implemented by the central idea that the drug molecules of interest (small chemicals or proteins) are subject to PK processes within the body described by distribution, metabolism and elimination routes in addition to the absorption mechanisms applied for small chemicals. By considering that these PK processes could be described mathematically, a number of differential equations have been applied to mechanistically express the underlying biological phenomena in a way that all body organs are linked through blood circulation. The suitable clinical validation of this approach then creates a framework where knowledge of clinical utility is generated from data covering in silico, in vitro and/or in vivo methodologies and experimentation upon new drug development. The main challenge still refers to the need for mutual understanding of 
both mathematics and biology from the users in order to avoid developing models which could be unrealistic or provide false results (106-108). Importantly in the case of nanomedicines, PBPK approaches are considered more complex based on the characteristics of the nanoparticles that make them different from small-molecule drugs and therapeutic proteins, since their PK profile is controlled by more multifaceted and interrelated relationships between the nanosystem and body physiology $(109,110)$. Nanostructured vehicles are designed either as solid nanoparticles consisting of polymers or inorganic materials or as liquid-based formulations that could be described as nanoemulsions. Such nanosystems are then loaded with small chemical drug molecules, therapeutic proteins, imaging probes or other relevant compounds (small RNA molecules, e.g. siRNAs/miRNAs) to cover a wide-range of medical uses from imaging to therapeutic applications (70,111-115). One of the driving forces for developing nanosystems for drug targeted delivery relies on their drug-loaded capacity for improved in vivo PK/PD profiles due to advanced metabolic stability and membrane permeability that could lead to improved bioavailability (for per os administration) and also to a prolonged pharmacological effect at the site of action with limited toxicity (116-118). Although these pharmacological advantages have intrigued the research on developing nanomedicines they also obstruct the straightforward application of PBPK approaches as they are applied today. This is due to several reasons which are also attributed to the characteristics of nanomedicines in order to obtain the above features of improved PK profiles. For example, it is easily conceivable that for the various systems of nanomedicines (i.e. dendrimers, nanocrystals, emulsions, liposomes, solid lipid nanoparticles, micelles and polymeric nanoparticles) different PBPK considerations should be implemented, since they represent different drugrelease systems. The latter also adds more complexity to the models, since for each one a different mechanism of drug release exists depending on the administration route - i.e. absorption through gut for per os delivery, release in the blood stream for intravenous infusion, or even release on the site of action for targeted administration. For example, in the typical setting of the PBPK approach, the controlled or sustained drug release systems are related with mechanisms of gut absorption (transporters, enzymes) or of other routes (i.e. skin, lungs) in order to predict the concentration of the drug that reaches the blood circulation which thereafter is used to further estimate the tissue concentrations, the PD profile on the site of action, and the elimination profiles (119-121). In contrast to nanomedicines, which act as drug nano-carriers, the controlled release often appears to be in the blood stream or in the site of action (i.e. tumor lesion, microenvironment) (122-126). These mechanisms add more complexity to the systems since additional parameters are involved, such as the mechanism of the release from the nanocarrier and the permeability into the site of action for each specific diseased tissue targeted, but also the drug's characteristic distribution such as solubility in plasma, plasma to tissue ratio and fraction of binding into tissue. In addition, the nanoparticle concentrations in systemic circulation and/or in the organ locally should be linked with drug release profiles related to either top-down or bottom-up PBPK models. The latter presents added complexity toward validation due to the difficulty to collect in vivo and clinical data for nanomedicines that act as carriers for targeted drug release. Taking into consideration the recent advancements in PGx approaches, then the use of PBPK modeling for nanomedicines also requires suitable adjustment to address drug-specific genetic PK/PD information. For example, issues that need to be addressed include the impact of polymorphisms of phase I and II drug metabolizing enzymes on the controlled/ sustained release over time of the drug from its nano-carrier into the systemic concentration (implying changes in the rate of the enzymatic reaction), since it is unclear whether it remains the same or even similar as for cases with classical sustained release systems for prolonged absorption $(120,127)$.

Based on the molecular knowledge accumulated thus far for epithelial-mesenchymal transition (EMT) and the known histological architecture of the tumor cells within the tissue, one can consider to build such a core PBPK model by suitably developing algorithms that incorporate parameters referring also to the metastatic behavior of malignant cells such as: i) cell adhesion molecule levels (e.g. for adherent junctions E-cadherin, for focal adhesion junctions integrin $\beta 1$, and for desmosomal junctions desmoglein-3); ii) the neovascularization extent (angiogenesis level) and the computationally depicted histological structure in the tumor area that could also contain measurable additional molecular markers involved in these processes [e.g. expression levels of vascular endothelial growth factor (VEGF), $\alpha v \beta 3$ integrin]. The clinical validation of any relevant adhesion molecule specification as metastatic biomarker, however, is needed for each tumor type, as recently shown in the case of oral squamous cell carcinoma (128). Finally, taking into consideration the known histological, genomic and cellular heterogeneity that exists within the tumor in various organs, one can further add information to specific components depending on the desired target diseased tissue (63). All the above parameters could contribute to the design of additional compartments (representing primary and metastatic tumor sites) although, to date, there are no published data available combining PBPK and metastatic tumors despite the fact that clinical trials are already executed for metastatic tumors and nanoparticles based on the preclinical data available (129).

Furthermore, much work exists for tumor growth modeling (usually for xerographs) and how they can possibly be coupled with PBPK approaches regarding the estimation of chemotherapy concentrations in tumor compartments and the improved adjustment of dosing regimen (83,130-132). Moreover, in addition to xenograft-based models, there is much effort toward the development of multi-scale in silico models aimed at the improved comprehension of tumor growth and the underlying mechanisms that lead to diverse outcomes of tumor lesions (133-141). Although both approaches sometimes require a tedious and time-consuming process due to the required individual extrapolation of data and validation of various factors contributing to cancer pathophysiology, diversity and modeling, they show encouraging results towards improved, personalized or stratified approaches to cancer treatment particularly for novel chemotherapeutic drug delivery schemes. The application of nanomedicines to prevent cancer metastasis provides new opportunities toward the development of improved therapies (142-146). It must also 
be emphasized that these approaches could be implemented at two levels of personalized approaches. The first level could be the stratification of patients based on primary and metastatic tumor regions and the second one the individual dosage regiments as proposed by in silico clinical trial models based on PBPK approaches. In addition, by successfully generating a metastatic behavior-based PBPK model, its application to assess the PK/PD profile of novel therapeutics very early in the drug developmental process may lead to improved productivity of drug candidate products with enhanced efficacy and clinical safety profiles, particularly in the more complex cases of metastatic tumors.

From the above mentioned, more focused efforts are needed toward establishing the optimum PK/PD correlations regarding the nanosystem physicochemical properties, the biological environment and the underlying molecular markers for balancing benefit-to-risk ratio in the preclinical/clinical development process. Drug-release kinetics either in systemic circulation or in the local diseased-tissue microenvironment (e.g. pulsatile vs. continuous release) is a crucial factor affecting the efficacy of nanomedicines and their safety behavior. It is thus urgent that a more thorough understanding of human physiology and pathophysiology and the interactions of biological systems with therapeutics be achieved. The latter implies a direction where the enhancement of pharmacological translational efficiency is facilitated through exploitation of the underlying developmental biology dynamics, the cellular diversity and the genomic heterogeneity for individual patients and for most illnesses. To successfully move on, the nanosystem formulation platforms, the pharmacological and preclinical assessment methodologies as well as the clinical development design must comply with such interdisciplinary research scheme requirements to readily overcome various obstructing parameters from bench to the clinic. Recently published work for cancer nanosystem platform methodologies and PBPK modeling add new insights to such a direction. In one study, the polymeric nanoparticle design platform was based on a combinatorial nature of simultaneously assessing their physicochemical diversity (size, surface hydrophilicity, targeting ligand density, drug loading and drug release) with the best clinical performance profiling for PK, biodistribution and efficacy upon encapsulation with chemotherapeutic agents (129). Importantly, the designed docetaxel-loaded polymeric nanoparticles targeted to the prostate-specific surface antigen exhibited similar PK, biodistribution, and safety profiles in three animal species (mice, rats and monkeys). In particular, their PK characteristics achieved much higher plasma concentrations of docetaxel for an extended duration that coincided with prolonged circulation in the vasculature and controlled release pattern in the body. To this end, docetaxel-loaded polymeric nanoparticles have been tested in humans with promising results confirming the previous data observed in animal species, whereas a phase II clinical trial is now running to evaluate their PK/PD behavior in patients with advanced metastatic prostate cancer (ClinicalTrials.gov Identifier: NCT01812746). In another similar study using the bisphosphonate ligand alendronate to target bone cancer lesions, the successful formulation of targeted polymeric nanoparticles for the controlled delivery of bortezomib to diminish off-target effects and increase tumor cell concentration was achieved (147). Bortezomib, a proteasome inhibitor, is clinically used for the therapy of multiple myeloma, a cancer primarily originating in bone marrow resulting in osteolytic lesions. Again, these bone microenvironment-targeted polymeric nanoparticles loaded with bortezomib have shown a favored PK/PD profile in terms of high retention, accumulation and bone homing capacity as well as enhanced survival and decreased tumor burden in mouse models of multiple myeloma. In a third study, the development of a PK model to quantify the effect of vascular physiology and permeability in individual patients for cancer nanotherapeutics was conducted (69). Notably, the specific particle size range of cancer nanosystems and the timed drug release profile were found to be crucial predictive rate-limiting formulation factors in order for the benefit-to-risk ratio to be maximized and clinically validated. The latter was correlated with the enhanced permeability and retention effect, attributed to the tumor microenvironment vascular pore size along with that exhibited for normal tissues, meaning that both normal physiology and pathophysiology of the vasculature contributed to the PK/PD cancer nanotherapeutic behavior. Unanimously, these results present evidence that the exploitation of PBPK modeling early in the development of cancer therapy nanomedicine represents the required solid ground and suitable framework for increasing the productivity later on in clinical phases and ensuring better efficacy and safety profiles for the respective marketed nanotherapeutics.

Based on the above, a proposed tumor-focused PBPK model for nanomedicines (PBPK-NM) is presented in Fig. 3. This vital need for successfully addressing the different PK behaviors of nanoparticles and thus nanomedicines in the body compared to that of their small-molecule drug counterparts, has already led the FDA to initiate projects such as 'PBPK Modeling of Nanomedicine' in order to apply computational modeling approaches to assess the safety of nano-scale materials. In particular, liposomal doxorubicin (Doxil), the first nanomedicine that entered the market and is used in the clinic for various solid tumors (ovarian cancer and myeloma) has been selected. The project launched in November 2014 is currently at the half of the road stage and it is expected to be concluded at the end of 2015 aimed at the development of computational approaches to be utilized to monitor Doxil in the blood and in different sites of the body (http://www.accessdata.fda.gov/FDATrack/trackproj?program=nctr\&id=NCTR-OSC-PM-PBPK-Modeling-ofNanomedicine).

\section{Future perspectives in advancing PBPK models to ensure practical clinical utility of cancer therapeutic interventions and PTx}

The predictive capacity of PBPK models allows them to also be successfully applied in assessing impacts on dose and particle size as well as on the in vivo performance of nanoformulations, either for medicines under development or for marketed drugs (148). However, fundamental issues exist that are hampering the advancement of personalized medicine and that highlight the lack of emerging predictive tools that could serve as a decision support mechanism for physicians to personalize treatment $(149,150)$. Particularly, this seems even more difficult for drug delivery by attempting to unify knowl- 


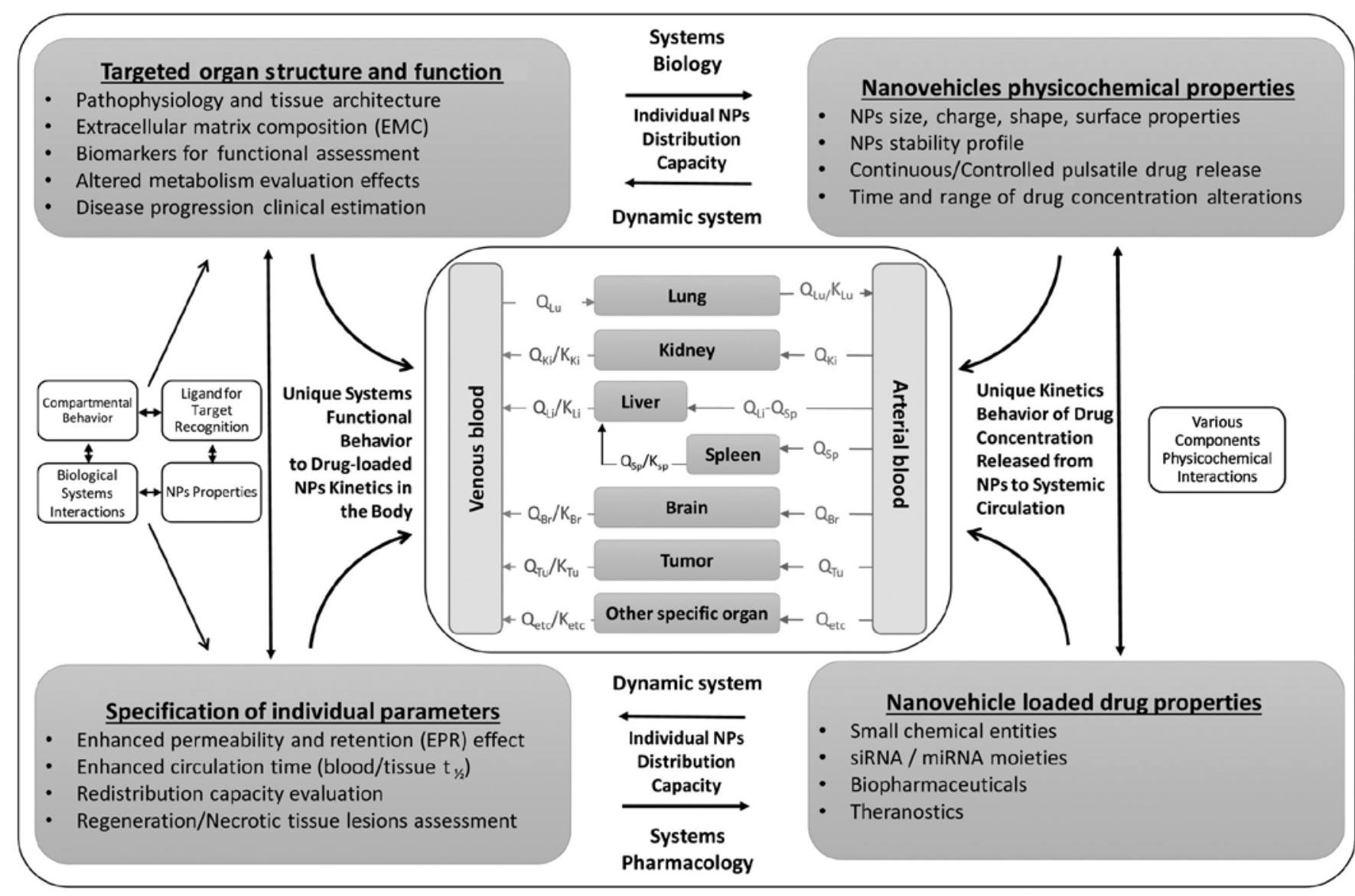

Figure 3. Diagrammatic illustration of a proposed population-based pharmacokinetic model for nanomedicines (PBPK-NM). The depiction of the complex and dynamic interactions between nanovehicle physicochemical behavior, loaded drug properties and specific architecture and function of the targeted organ shown is adapted for tumor therapy. In particular, the two crucial components in the PBPK-NM model represent: i) the system dynamics (systems biology), i.e. the interaction between the targeted organ structure and function and nanovehicle properties; and ii) the pharmacological dynamics (systems pharmacology), i.e. the loaded drug properties and the specific organ parameters contributing to the PK/PD outcome. These two component interactions modulate the unique behavior of systems to drug-loaded NP kinetics in the body, as well as the unique kinetic behavior of the drug concentration released from NPs to the systemic circulation. The latter can lead to an individual distribution capacity for the drug-loaded NPs and a unique system functional behavior to it, which definitely enables a strong potential for stratified and personalized treatment. Q, The blood flow in the various organs; $\mathrm{K}$, the partition coefficient of the loaded drug for a specific organ with $\mathrm{Lu}, \mathrm{Ki}, \mathrm{Li}, \mathrm{Sp}, \mathrm{Br}$, Tu, representing lung, kidney, liver, spleen, brain and tumor, respectively.

edge from heterogeneous data derived e.g. from personal genome sequencing, structural, functional and chemical genomics applications, as well as the PK/PD drug profiles including the clinical setting. In contrast, PBPK models represent platforms where organ- and tissue-specific parameters, genomic variation data and physicochemical drug properties can be efficiently inter-correlated to allow the prediction of PK/PD drug behavior in the body for specific populations or individual patients. Importantly, the enrichment of the predictive capacity of PBPK models towards empowering individualized drug response phenotype decisions could be achieved through incorporation of: i) genome-wide drugtarget molecular interactions; ii) temporal and spatial scale macromolecular conformation state behavior; iii) drug-driven molecular circuit pathways; and iv) in real-time monitoring the impact of environmental and clinical parameters. To this end, the structure-enabled integrative modeling to effectively predict QT interval prolongation and minimize the drug-induced arrhythmia profile of delivered medicines in the clinical setting has been recently exemplified (150). The latter implements crucial clinical information related to molecular, genomics, pharmacological and chemical drug- related aspects including those of pharmacovigilance that have been previously established in regulatory legislation for the arrhythmogenic behavior of drugs (8).

As far as drug delivery is concerned, new tools are tilting health-care control from physicians to patients with the paradigm of the delivery of insulin using telemedicine (151). Three dimensional (3D) printing technologies emerge as an innovative approach to design pharmaceutical dosage forms for personalized medicine (152). Recently, 3D printing was used for the production of tablets capable of satisfying regulatory tests and matching the release of standard commercial tablets (153). Remote controlled capsules (RCCs) have been extensively used in the field of site-specific drug delivery using micro-electronic mechanical system (MEMS) technology 154). MEMS combined with medical devices (e.g. contact lenses) can be used for ocular diagnostics (155). Finally dosing devices and solid dosage forms might allow oral individual drug therapy (156).

The availability of information science technologies aimed to develop advanced tools and databases for electronic health records for patients raise the question of whether virtual population profiles gained from epidemiologic 
recorded data with data generated from the patients and/ or hospital driven databases and relative software could match. The latter, if occurred, would permit the fitting of modeled data of virtual profiles with profiles of real life patients and thus initiate personalized or stratified medicine approaches. Moreover, it will also advance the next step for the development of unique decision-making tools or for novel approaches during R\&D but also for PTx procedures. However, the absence of a roadmap on how the regulatory environment in drug development and healthcare is gaining major clinical benefit and economic value from personalized medicine applications has clearly restricted the required evidence to informed decision-making and assessment of genomic priorities (42). It is therefore of important priority to work and present evidence by establishing infrastructure and methodologies capable of confirming cost-effectiveness in addition to clinical effectiveness in a robust and timely manner for decision makers (43); and by doing so, the parallel advancement of PBPK models will empower the translation capacity and practical clinical utility of personalized medicine decisions, thus benefitting healthcare and stabilizing PTx worldwide (157). These new technologies are expected to revolutionize existing healthcare and drug delivery, in a way to enrich personalized medicine capabilities and precision for broader clinical utility of translational medicine therapeutic interventions worldwide.

\section{References}

1. Braeckmans K, De Smedt SC, Leblans M, Pauwels R and Demeester J: Encoding microcarriers: Present and future technologies. Nat Rev Drug Discov 1: 447-456, 2002.

2. Ginsburg GS and Willard HF: Genomic and personalized medicine: Foundations and applications. Transl Res 154: 277-287, 2009.

3. Hertz DL and McLeod HL: Use of pharmacogenetics for predicting cancer prognosis and treatment exposure, response and toxicity. J Hum Genet 58: 346-352, 2013.

4. Kaddurah-Daouk R and Weinshilboum RM; Pharmacometabolomics Research Network: Pharmacometabolomics: Implications for clinical pharmacology and systems pharmacology. Clin Pharmacol Ther 95: 154-167, 2014.

5. Roses AD, Saunders AM, Lutz MW, Zhang N, Hariri AR, Asin KE, Crenshaw DG, Budur K, Burns DK and Brannan SK: New applications of disease genetics and pharmacogenetics to drug development. Curr Opin Pharmacol 14: 81-89, 2014.

6. Vizirianakis IS: Challenges in current drug delivery from the potential application of pharmacogenomics and personalized medicine in clinical practice. Curr Drug Deliv 1: 73-80, 2004.

7. Vizirianakis IS: Improving pharmacotherapy outcomes by pharmacogenomics: From expectation to reality? Pharmacogenomics 6: 701-711, 2005

8. Vizirianakis IS: Clinical translation of genotyping and haplotyping data: Implementation of in vivo pharmacology experience leading drug prescription to pharmacotyping. Clin Pharmacokinet 46: 807-824, 2007.

9. Vizirianakis IS: Advancement of pharmacogenomics toward pharmacotyping in drug prescription: Concepts, challenges, and perspectives for personalized medicine. In: Handbook of Personalized Medicine: Advances in Nanotechnology, Drug Delivery and Therapy. Vizirianakis IS (ed). Pan Stanford Publishing, Singapore, pp893-952, 2014.

10. Pirmohamed M: Personalized pharmacogenomics: Predicting efficacy and adverse drug reactions. Annu Rev Genomics Hum Genet 15: 349-370, 2014.

11. Ahn K, Luo J, Berg A, Keefe D and Wu R: Functional mapping of drug response with pharmacodynamic-pharmacokinetic principles. Trends Pharmacol Sci 31: 306-311, 2010.

12. Daka A and Peer D: RNAi-based nanomedicines for targeted personalized therapy. Adv Drug Deliv Rev 64: 1508-1521, 2012.
13. Debbage P: Targeted drugs and nanomedicine: Present and future. Curr Pharm Des 15: 153-172, 2009.

14. Huttenhower $\mathrm{C}$ and Hofmann O: A quick guide to large-scale genomic data mining. PLOS Comput Biol 6: e1000779, 2010.

15. Janowski M, Bulte JW and Walczak P: Personalized nanomedicine advancements for stem cell tracking. Adv Drug Deliv Rev 64: 1488-1507, 2012.

16. Mura $S$ and Couvreur P: Nanotheranostics for personalized medicine. Adv Drug Deliv Rev 64: 1394-1416, 2012.

17. Petersen AL, Hansen AE, Gabizon A and Andresen TL: Liposome imaging agents in personalized medicine. Adv Drug Deliv Rev 64: 1417-1435, 2012.

18. Ryu JH, Koo H, Sun IC, Yuk SH, Choi K, Kim K and Kwon IC: Tumor-targeting multi-functional nanoparticles for theragnosis: New paradigm for cancer therapy. Adv Drug Deliv Rev 64: 1447-1458, 2012

19. Vizirianakis IS, Chatzopoulou M, Bonovolias ID, Nicolaou I, Demopoulos VJ and Tsiftsoglou AS: Toward the development of innovative bifunctional agents to induce differentiation and to promote apoptosis in leukemia: Clinical candidates and perspectives. J Med Chem 53: 6779-6810, 2010.

20. Wieland $\mathrm{M}$ and Fussenegger M: Reprogrammed cell delivery for personalized medicine. Adv Drug Deliv Rev 64: 1477-1487, 2012.

21. Cook D, Brown D, Alexander R, March R, Morgan P, Satterthwaite $G$ and Pangalos MN: Lessons learned from the fate of AstraZeneca's drug pipeline: A five-dimensional framework. Nat Rev Drug Discov 13: 419-431, 2014.

22. Cree IA: Designing personalised cancer treatments. J Control Release 172: 405-409, 2013.

23. Hillgren KM, Keppler D, Zur AA, Giacomini KM, Stieger B, Cass CE and Zhang L; International Transporter Consortium: Emerging transporters of clinical importance: An update from the International Transporter Consortium. Clin Pharmacol Ther 94: 52-63, 2013.

24. Huang M, Shen A, Ding J and Geng M: Molecularly targeted cancer therapy: Some lessons from the past decade. Trends Pharmacol Sci 35: 41-50, 2014.

25. Ingelman-Sundberg M: Pharmacogenetics of cytochrome P450 and its applications in drug therapy: The past, present and future. Trends Pharmacol Sci 25: 193-200, 2004.

26. Jain KK: Innovative diagnostic technologies and their significance for personalized medicine. Mol Diagn Ther 14: 141-147, 2010.

27. Lee JW, Aminkeng F, Bhavsar AP, Shaw K, Carleton BC, Hayden MR and Ross CJ: The emerging era of pharmacogenomics: Current successes, future potential, and challenges. Clin Genet 86: 21-28, 2014

28. Ntziachristos V and Razansky D: Molecular imaging by means of multispectral optoacoustic tomography (MSOT). Chem Rev 110: 2783-2794, 2010.

29. Ntziachristos V, Schellenberger EA, Ripoll J, Yessayan D, Graves E, Bogdanov A Jr, Josephson L and Weissleder R: Visualization of antitumor treatment by means of fluorescence molecular tomography with an annexin V-Cy5.5 conjugate. Proc Natl Acad Sci USA 101: 12294-12299, 2004.

30. Pirmohamed M: Pharmacogenetics: Past, present and future. Drug Discov Today 16: 852-861, 2011.

31. Sadee W: Pharmacogenomic biomarkers: Validation needed for both the molecular genetic mechanism and clinical effect. Pharmacogenomics 12: 675-680, 2011.

32. Vizirianakis IS: Nanomedicine and personalized medicine toward the application of pharmacotyping in clinical practice to improve drug-delivery outcomes. Nanomedicine 7: 11-17, 2011.

33. Flowers CR and Veenstra D: The role of cost-effectiveness analysis in the era of pharmacogenomics. Pharmacoeconomics 22: 481-493, 2004.

34. Payne K and Shabaruddin FH: Cost-effectiveness analysis in pharmacogenomics. Pharmacogenomics 11: 643-646, 2010

35. Sorich MJ, Wiese MD and Pekarsky B: Cost-effectiveness of genotyping to guide treatment. Pharmacogenomics 15: 727-729, 2014.

36. Wong WB, Carlson JJ, Thariani R and Veenstra DL: Cost effectiveness of pharmacogenomics: A critical and systematic review. Pharmacoeconomics 28: 1001-1013, 2010.

37. Wu AHB, Babic $\mathrm{N}$ and Yeo KT: Implementation of pharmacogenomics into the clinical practice of therapeutics: Issues for the clinician and the laboratorian. Per Med 6: 315-327, 2009.

38. Fleeman N, McLeod C, Bagust A, Beale S, Boland A, Dundar Y, Jorgensen A, Payne K, Pirmohamed M, Pushpakom S, et al: The clinical effectiveness and cost-effectiveness of testing for cytochrome $\mathrm{P} 450$ polymorphisms in patients with schizophrenia treated with antipsychotics: A systematic review and economic evaluation. Health Technol Assess 14: 1-157, iii, 2010. 
39. Gurwitz D, Rodríguez-Antona C, Payne K, Newman W, Gisbert JP, de Mesa EG and Ibarreta D: Improving pharmacovigilance in Europe: TPMT genotyping and phenotyping in the UK and Spain. Eur J Hum Genet 17: 991-998, 2009.

40. Thompson AJ, Newman WG, Elliott RA, Roberts SA, Tricker K and Payne K: The cost-effectiveness of a pharmacogenetic test: A trial-based evaluation of TPMT genotyping for azathioprine. Value Health 17: 22-33, 2014

41. van den Akker-van Marle ME, Gurwitz D, Detmar SB, Enzing CM, Hopkins MM, Gutierrez de Mesa E and Ibarreta D: Cost-effectiveness of pharmacogenomics in clinical practice: A case study of thiopurine methyltransferase genotyping in acute lymphoblastic leukemia in Europe. Pharmacogenomics 7: 783-792, 2006

42. Phillips KA, Ann Sakowski J, Trosman J, Douglas MP, Liang SY and Neumann P: The economic value of personalized medicine tests: Genet Med 16: 251-257, 2014.

43. Shabaruddin FH and Payne K: Evaluating the cost-effectiveness of pharmacogenomics in clinical practice. In: Handbook of Personalized Medicine: Advances in Nanotechnology, Drug Delivery and Therapy. Vizirianakis IS (ed). Pan Stanford Publishing, Singapore, pp779-812, 2014.

44. Poulin P, Jones RD, Jones HM, Gibson CR, Rowland M, Chien JY, Ring BJ, Adkison KK, Ku MS, He H, et al: PHRMA CPCDC initiative on predictive models of human pharmacokinetics, part 5: Prediction of plasma concentration-time profiles in human using the physiologically-based pharmacokinetic modeling approach. J Pharm Sci 100: 4127-4157, 2011.

45. Ring BJ, Chien JY, Adkison KK, Jones HM, Rowland M, Jones RD, Yates JW, Ku MS, Gibson CR, He H, et al: PhRMA CPCDC initiative on predictive models of human pharmacokinetics, part 3: Comparative assessement of prediction methods of human clearance. J Pharm Sci 100: 4090-4110, 2011.

46. Bates S: Progress towards personalized medicine. Drug Discov Today 15: 115-120, 2010.

47. Fu G, Liu J, Luo J, Zhong W, Wang Y, Wang N and Wu R: Systems mapping: A computational tool for personalized medicine. In: Handbook of Personalized Medicine: Advances in Nanotechnology, Drug Delivery and Therapy. Vizirianakis IS (ed). Pan Stanford Publishing, Singapore, pp321-340, 2014

48. Wu R, Tong C, Wang Z, Mauger D, Tantisira K, Szefler SJ, Chinchilli VM and Israel E: A conceptual framework for pharmacodynamic genome-wide association studies in pharmacogenomics. Drug Discov Today 16: 884-890, 2011.

49. Hong H, Perkins R, Shi L, Fang H, Mendrick DL and Tong W: Molecular biomarkers for personalized medicine. In: Handbook of Personalized Medicine: Advances in Nanotechnology, Drug Delivery and Therspy. Vizirianakis IS (ed). Pan Stanford Publishing, Singapore, pp607-644, 2014.

50. Gonzalez de Castro D, Clarke PA, Al-Lazikani B and Workman P Personalized cancer medicine: Molecular diagnostics, predictive biomarkers, and drug resistance. Clin Pharmacol Ther 93 252-259, 2013

51. Yap TA, Sandhu SK, Workman P and de Bono JS: Envisioning the future of early anticancer drug development. Nat Rev Cancer 10 514-523, 2010

52. Rubin EH and Gilliland DG: Drug development and clinical trials - the path to an approved cancer drug. Nat Rev Clin Oncol 9: 215-222, 2012.

53. Vizirianakis IS and Fatouros DG: Personalized nanomedicine: Paving the way to the practical clinical utility of genomics and nanotechnology advancements. Adv Drug Deliv Rev 64: 1359-1362, 2012

54. Vizirianakis IS: Handbook of Personalized Medicine: Advances in Nanotechnology, Drug Delivery, and Therapy. Pan Stanford Publishing, Singapore, 2014

55. Swanson TW, Akkari PA, Arbuckle JB, Grossman I, Sundseth SS and Roses AD: Methodology to enable integration of genomic knowledge into drug development. In: Handbook of Personalized Medicine: Advances in Nanotechnology, Drug Delivery and Therpy. Vizirianakis IS (ed). Pan Stanford Publishing, Singapore, pp645-684, 2014.

56. Jamei M, Rowland YK and Rostami-Hodjegan A: Framework, organization, and applications of the Simcyp population-based simulator to support new drug development. In: Handbook of Personalized Medicine: Advances in Nanotechnology, Drug Delivery and Therapy. Vizirianakis IS (ed). Pan Stanford Publishing, Singapore, pp685-726. Pan Stanford Publishing, Singapore, 2014

57. Amstutz U and Carleton BC: Pharmacogenetic testing: Time for clinical practice guidelines. Clin Pharmacol Ther 89: 924-927, 2011.
58. Johnson JA, Gong L, Whirl-Carrillo M, Gage BF, Scott SA, Stein CM, Anderson JL, Kimmel SE, Lee MT, Pirmohamed M, et al; Clinical Pharmacogenetics Implementation Consortium: Clinical Pharmacogenetics Implementation Consortium Guidelines for $C Y P 2 C 9$ and $V K O R C 1$ genotypes and warfarin dosing. Clin Pharmacol Ther 90: 625-629, 2011.

59. Relling MV, Gardner EE, Sandborn WJ, Schmiegelow K, Pui CH, Yee SW, Stein CM, Carrillo M, Evans WE and Klein TE; Clinical Pharmacogenetics Implementation Consortium: Clinical Pharmacogenetics Implementation Consortium guidelines for thiopurine methyltransferase genotype and thiopurine dosing. Clin Pharmacol Ther 89: 387-391, 2011.

60. Relling MV and Klein TE: CPIC: Clinical Pharmacogenetics Implementation Consortium of the Pharmacogenomics Research Network. Clin Pharmacol Ther 89: 464-467, 2011.

61. Scott SA, Sangkuhl K, Gardner EE, Stein CM, Hulot JS Johnson JA, Roden DM, Klein TE and Shuldiner AR; Clinical Pharmacogenetics Implementation Consortium: Clinical Pharmacogenetics Implementation Consortium guidelines for cytochrome P450-2C19 (CYP2C19) genotype and clopidogrel therapy. Clin Pharmacol Ther 90: 328-332, 2011.

62. Swen JJ, Nijenhuis M, de Boer A, Grandia L, Maitland-van der Zee AH, Mulder H, Rongen GA, van Schaik RH, Schalekamp T, Touw DJ, et al: Pharmacogenetics: From bench to byte - an update of guidelines. Clin Pharmacol Ther 89: 662-673, 2011.

63. Hanahan D and Weinberg RA: Hallmarks of cancer: The next generation. Cell 144: 646-674, 2011.

64. Workman P: The opportunities and challenges of personalized genome-based molecular therapies for cancer: Targets, technologies, and molecular chaperones. Cancer Chemother 52 (Suppl 1): S45-S56, 2003.

65. Jain RK: Normalizing tumor microenvironment to treat cancer: Bench to bedside to biomarkers. J Clin Oncol 31: 2205-2218, 2013.

66. Khawar IA, Kim JH and Kuh HJ: Improving drug delivery to solid tumors: J Control Release 201: 78-89, 2015.

67. Scott JG, Hjelmeland AB, Chinnaiyan P, Anderson AR and Basanta D: Microenvironmental variables must influence intrinsic phenotypic parameters of cancer stem cells to affect tumourigenicity. PLOS Comput Biol 10: e1003433, 2014.

68. Watnick RS: The role of the tumor microenvironment in regulating angiogenesis. Cold Spring Harb Perspect Med 2: a006676, 2012.

69. Kirtane AR, Siegel RA and Panyam J: A pharmacokinetic model for quantifying the effect of vascular permeability on the choice of drug carrier: A framework for personalized nanomedicine. J Pharm Sci 104: 1174-1186, 2015.

70. Moss DM and Siccardi M: Optimizing nanomedicine pharmacokinetics using physiologically based pharmacokinetics modelling. Br J Pharmacol 171: 3963-3979, 2014

71. Andre F, Mardis E, Salm M, Soria JC, Siu LL and Swanton C: Prioritizing targets for precision cancer medicine. Ann Oncol 25: 2295-2303, 2014

72. Collins FS and Varmus H: A new initiative on precision medicine. N Engl J Med 372: 793-795, 2015.

73. Doudican NA, Kumar A, Singh NK, Nair PR, Lala DA, Basu K, Talawdekar AA, Sultana Z, Tiwari KK, Tyagi A, et al: Personalization of cancer treatment using predictive simulation. J Transl Med 13: 43, 2015.

74. Roychowdhury S and Chinnaiyan AM: Translating genomics for precision cancer medicine. Annu Rev Genomics Hum Genet 15: 395-415, 2014

75. Binkhorst L, Mathijssen RH, Jager A and van Gelder T: Individualization of tamoxifen therapy: Much more than just CYP2D6 genotyping. Cancer Treat Rev 41: 289-299, 2015.

76. Smith GL: The long and short of tamoxifen therapy: A review of the ATLAS trial. J Adv Pract Oncol 5: 57-60, 2014.

77. Borges S, Desta Z, Li L, Skaar TC, Ward BA, Nguyen A, Jin Y, Storniolo AM, Nikoloff DM, Wu L, et al: Quantitative effect of $C Y P 2 D 6$ genotype and inhibitors on tamoxifen metabolism: Implication for optimization of breast cancer treatment. Clin Pharmacol Ther 80: 61-74, 2006.

78. Flockhart D: CYP2D6 genotyping and the pharmacogenetics of tamoxifen. Clin Adv Hematol Oncol 6: 493-494, 2008.

79. Jin Y, Desta Z, Stearns V, Ward B, Ho H, Lee KH, Skaar T, Storniolo AM, Li L, Araba A, et al: CYP2D6 genotype, antidepressant use, and tamoxifen metabolism during adjuvant breast cancer treatment. J Natl Cancer Inst 97: 30-39, 2005.

80. Stearns V, Johnson MD, Rae JM, Morocho A, Novielli A, Bhargava P, Hayes DF, Desta Z and Flockhart DA: Active tamoxifen metabolite plasma concentrations after coadministration of tamoxifen and the selective serotonin reuptake inhibitor paroxetine. J Natl Cancer Inst 95: 1758-1764, 2003. 
81. Dickschen K, Willmann S, Thelen K, Lippert J, Hempel G and Eissing T: Physiologically based pharmacokinetic modeling of tamoxifen and its metabolites in women of different CYP2D6 phenotypes provides new insight into the tamoxifen mass balance. Front Pharmacol 3: 92, 2012.

82. Drbohlavova J, Chomoucka J, Adam V, Ryvolova M, Eckschlager T, Hubalek J and Kizek R: Nanocarriers for anticancer drugs - new trends in nanomedicine. Curr Drug Metab 14: $547-564,2013$

83. Ballesta A, Zhou Q, Zhang X, Lv H and Gallo JM: Multiscale design of cell-type-specific pharmacokinetic/pharmacodynamic models for personalized medicine: Application to temozolomide in brain tumors. CPT Pharmacometrics Syst Pharmacol 3: e112, 2014

84. Block M: Physiologically based pharmacokinetic and pharmacodynamic modeling in cancer drug development: Status, potential and gaps. Expert Opin Drug Metab Toxicol 11: 743-756, 2015.

85. Upreti M, Jyoti A and Sethi P: Tumor microenvironment and nanotherapeutics. Transl Cancer Res 2: 309-319, 2013.

86. Maji R, Dey NS, Satapathy BS, Mukherjee B and Mondal S: Preparation and characterization of Tamoxifen citrate loaded nanoparticles for breast cancer therapy. Int J Nanomedicine 9 3107-3118, 2014

87. Pandey SK, Ghosh S, Maiti P and Haldar C: Therapeutic efficacy and toxicity of tamoxifen loaded PLA nanoparticles for breas cancer. Int J Biol Macromol 72: 309-319, 2015.

88. Hersh EM, O'Day SJ, Ribas A, Samlowski WE, Gordon MS Shechter DE, Clawson AA and Gonzalez R: A phase 2 clinical trial of $n a b$-paclitaxel in previously treated and chemotherapynaive patients with metastatic melanoma. Cancer 116: 155-163, 2010

89. Jehn CF, Boulikas T, Kourvetaris A, Kofla G, Possinger K and Lüftner D: First safety and response results of a randomized phase III study with liposomal platin in the treatment of advanced squamous cell carcinoma of the head and neck (SCCHN). Anticancer Res 28: 3961-3964, 2008.

90. Mamot C, Ritschard R, Wicki A, Stehle G, Dieterle T, Bubendorf L, Hilker C, Deuster S, Herrmann R and Rochlitz C: Tolerability, safety, pharmacokinetics, and efficacy of doxorubicin-loaded anti-EGFR immunoliposomes in advanced solid tumours: A phase 1 dose-escalation study. Lancet Oncol 13 1234-1241, 2012.

91. Zhao P and Astruc D: Docetaxel nanotechnology in anticancer therapy. ChemMedChem 7: 952-972, 2012

92. Baish JW, Stylianopoulos T, Lanning RM, Kamoun WS, Fukumura D, Munn LL and Jain RK: Scaling rules for diffusive drug delivery in tumor and normal tissues. Proc Natl Acad Sci USA 108: 1799-1803, 2011.

93. Bachler G, von Goetz N and Hungerbühler K: A physiologically based pharmacokinetic model for ionic silver and silver nanoparticles. Int J Nanomedicine 8: 3365-3382, 2013.

94. Bachler G, von Goetz N and Hungerbuhler K: Using physiologically based pharmacokinetic (PBPK) modeling for dietary risk assessment of titanium dioxide $\left(\mathrm{TiO}_{2}\right)$ nanoparticles. Nanotoxicology 9: 373-380, 2015.

95. Liu J, Zheng X, Yan L, Zhou L, Tian G, Yin W, Wang L, Liu Y, $\mathrm{Hu} \mathrm{Z}$, Gu Z, et al: Bismuth sulfide nanorods as a precision nanomedicine for in vivo multimodal imaging-guided phototherma therapy of tumor. ACS Nano 9: 696-707, 2015

96. Mouffouk F, Simão T, Dornelles DF, Lopes AD, Sau P, Martins J, Abu-Salah KM, Alrokayan SA, Rosa da Costa AM and dos Santos NR: Self-assembled polymeric nanoparticles as new, smart contrast agents for cancer early detection using magnetic resonance imaging. Int J Nanomedicine 10: 63-76, 2015.

97. Perera RH, Hernandez C, Zhou H, Kota P, Burke A and Exner AA: Ultrasound imaging beyond the vasculature with new generation contrast agents. Wiley Interdiscip Rev Nanomed Nanobiotechnol 7: 593-608, 2015.

98. Torchilin VP: Multifunctional, stimuli-sensitive nanoparticulate systems for drug delivery. Nat Rev Drug Discov 13: 813-827, 2014

99. Barrett JS, Della Casa Alberighi O, Läer S and Meibohm B: Physiologically based pharmacokinetic (PBPK) modeling in children. Clin Pharmacol Ther 92: 40-49, 2012.

100. Chetty M, Li L, Rose R, Machavaram K, Jamei M, RostamiHodjegan A and Gardner I: Prediction of the pharmacokinetics, pharmacodynamics, and efficacy of a monoclonal antibody, using a physiologically based pharmacokinetic FcRn model. Front Immunol 5: 670, 2014
101. Diao L and Meibohm B: Pharmacokinetics and pharmacokinetic-pharmacodynamic correlations of therapeutic peptides. Clin Pharmacokinet 52: 855-868, 2013.

102. Dostalek M, Gardner I, Gurbaxani BM, Rose RH and Chetty M: Pharmacokinetics, pharmacodynamics and physiologicallybased pharmacokinetic modelling of monoclonal antibodies. Clin Pharmacokinet 52: 83-124, 2013.

103. Jones HM, Chen Y, Gibson C, Heimbach T, Parrott N, Peters SA, Snoeys J, Upreti VV, Zheng M and Hall SD: Physiologically based pharmacokinetic modeling in drug discovery and development: A pharmaceutical industry perspective. Clin Pharmacol Ther 97: 247-262, 2015.

104. Dranitsaris G, Amir E and Dorward K: Biosimilars of biological drug therapies: Regulatory, clinical and commercial considerations. Drugs 71: 1527-1536, 2011.

105. Wang J, Iyer S, Fielder PJ, Davis JD and Deng R: Projecting human pharmacokinetics of monoclonal antibodies from nonclinical data: Comparative evaluation of prediction approaches in early drug development. Biopharm Drug Dispos: Apr 13, 2015 (Epub ahead of print).

106. Bouzom F, Ball K, Perdaems N and Walther B: Physiologically based pharmacokinetic (PBPK) modelling tools: How to fit with our needs? Biopharm Drug Dispos 33: 55-71, 2012.

107. Nyberg J, Bazzoli C, Ogungbenro K, Aliev A, Leonov S, Duffull S, Hooker AC and Mentré F: Methods and software tools for design evaluation in population pharmacokineticspharmacodynamics studies. Br J Clin Pharmacol 79: 6-17, 2015

108. Rowland M, Peck C and Tucker G: Physiologically-based pharmacokinetics in drug development and regulatory science. Annu Rev Pharmacol Toxicol 51: 45-73, 2011

109. Moghimi SM, Hunter AC and Andresen TL: Factors controlling nanoparticle pharmacokinetics: An integrated analysis and perspective. Annu Rev Pharmacol Toxicol 52: 481-503, 2012.

110. Zhang XQ, Xu X, Bertrand N, Pridgen E, Swami A and Farokhzad OC: Interactions of nanomaterials and biological systems: Implications to personalized nanomedicine. Adv Drug Deliv Rev 64: 1363-1384, 2012.

111. Davis ME, Zuckerman JE, Choi CH, Seligson D, Tolcher A, Alabi CA, Yen Y, Heidel JD and Ribas A: Evidence of RNAi in humans from systemically administered siRNA via targeted nanoparticles. Nature 464: 1067-1070, 2010.

112. Gao W, Xiao Z, Radovic-Moreno A, Shi J, Langer R and Farokhzad OC: Progress in siRNA delivery using multifunctional nanoparticles. Methods Mol Biol 629: 53-67, 2010.

113. Zhou J, Shum KT, Burnett JC and Rossi JJ: Nanoparticlebased delivery of RNAi therapeutics: Progress and challenges. Pharmaceuticals 6: 85-107, 2013.

114. Abakumov MA, Nukolova NV, Sokolsky-Papkov M, Shein SA, Sandalova TO, Vishwasrao HM, Grinenko NF, Gubsky IL, Abakumov AM, Kabanov AV, et al: VEGF-targeted magnetic nanoparticles for MRI visualization of brain tumor. Nanomedicine 11: 825-833, 2015

115. Yohan D and Chithrani BD: Applications of nanoparticles in nanomedicine. J Biomed Nanotechnol 10: 2371-2392, 2014.

116. Pridgen EM, Alexis F and Farokhzad OC: Polymeric nanoparticle technologies for oral drug delivery. Clin Gastroenterol Hepatol 12: 1605-1610, 2014

117. Wright J: Deliver on a promise. Sci Am 311: S12-S13, 2014.

118. Zhang L, Gu FX, Chan JM, Wang AZ, Langer RS and Farokhzad OC: Nanoparticles in medicine: Therapeutic applications and developments. Clin Pharmacol Ther 83: 761-769, 2008.

119. Aoyama T, Omori T, Watabe S, Shioya A, Ueno T, Fukuda N and Matsumoto Y: Pharmacokinetic/pharmacodynamic modeling and simulation of rosuvastatin using an extension of the indirect response model by incorporating a circadian rhythm. Biol Pharm Bull 33: 1082-1087, 2010.

120. Chetty M, Rose RH, Abduljalil K, Patel N, Lu G, Cain T, Jamei $\mathrm{M}$ and Rostami-Hodjegan A: Applications of linking PBPK and PD models to predict the impact of genotypic variability, formulation differences, differences in target binding capacity and target site drug concentrations on drug responses and variability. Front Pharmacol 5: 258, 2014.

121. Rose RH, Neuhoff S, Abduljalil K, Chetty M, Rostami-Hodjegan A and Jamei M: Application of a physiologically based pharmacokinetic model to predict $O A T P I B 1$-related variability in pharmacodynamics of rosuvastatin. CPT Pharmacometrics Syst Pharmacol 3: e124, 2014. 
122. Abbad S, Wang C, Waddad AY, Lv H and Zhou J: Preparation, in vitro and in vivo evaluation of polymeric nanoparticles based on hyaluronic acid-poly(butyl cyanoacrylate) and D-alphatocopheryl polyethylene glycol 1000 succinate for tumor-targeted delivery of morin hydrate. Int J Nanomedicine 10: 305-320, 2015.

123. Abouzeid AH, Patel NR, Sarisozen C and Torchilin VP: Transferrin-targeted polymeric micelles co-loaded with curcumin and paclitaxel: Efficient killing of paclitaxel-resistant cancer cells. Pharm Res 31: 1938-1945, 2014.

124. Acharya S and Sahoo SK: PLGA nanoparticles containing various anticancer agents and tumour delivery by EPR effect. Adv Drug Deliv Rev 63: 170-183, 2011.

125. Chauhan VP, Stylianopoulos T, Martin JD, Popović Z, Chen O, Kamoun WS, Bawendi MG, Fukumura D and Jain RK: Normalization of tumour blood vessels improves the delivery of nanomedicines in a size-dependent manner. Nat Nanotechnol 7: 383-388, 2012.

126. Jain RK and Stylianopoulos T: Delivering nanomedicine to solid tumors. Nat Rev Clin Oncol 7: 653-664, 2010.

127. Olivares-Morales A, Kamiyama Y, Darwich AS, Aarons L and Rostami-Hodjegan A: Analysis of the impact of controlled release formulations on oral drug absorption, gut wall metabolism and relative bioavailability of CYP3A substrates using a physiologically-based pharmacokinetic model. Eur J Pharm Sci 67: 32-44, 2015.

128. Kyrodimou M, Andreadis D, Drougou A, Amanatiadou EP, Angelis L, Barbatis C, Epivatianos A and Vizirianakis IS: Desmoglein- $3 / \gamma$-catenin and E-cadherin $/ \beta$-catenin differential expression in oral leukoplakia and squamous cell carcinoma. Clin Oral Investig 18: 199-210, 2014.

129. Hrkach J, Von Hoff D, Mukkaram Ali M, Andrianova E, Auer J, Campbell T, De Witt D, Figa M, Figueiredo M, Horhota A, et al: Preclinical development and clinical translation of a PSMA-targeted docetaxel nanoparticle with a differentiated pharmacological profile. Sci Transl Med 4: 128ra39, 2012.

130. Hudachek SF and Gustafson DL: Physiologically based pharmacokinetic model of lapatinib developed in mice and scaled to humans. J Pharmacokinet Pharmacodyn 40: 157-176, 2013.

131. Poulin P, Hop CE, Salphati L and Liederer BM: Correlation of tissue-plasma partition coefficients between normal tissues and subcutaneous xenografts of human tumor cell lines in mouse as a prediction tool of drug penetration in tumors. J Pharm Sci 102: 1355-1369, 2013.

132. Zhou Q, Guo P, Kruh GD, Vicini P, Wang X and Gallo JM: Predicting human tumor drug concentrations from a preclinical pharmacokinetic model of temozolomide brain disposition. Clin Cancer Res 13: 4271-4279, 2007.

133. Castorina P, Carcò D, Guiot C and Deisboeck TS: Tumor growth instability and its implications for chemotherapy. Cancer Res 69 : 8507-8515, 2009.

134. Ganguly R and Puri IK: Mathematical model for chemotherapeutic drug efficacy in arresting tumour growth based on the cancer stem cell hypothesis. Cell Prolif 40: 338-354, 2007.

135. Hubbard ME and Byrne HM: Multiphase modelling of vascular tumour growth in two spatial dimensions. J Theor Biol 316 70-89, 2013

136. Johnson D, McKeever S, Stamatakos G, Dionysiou D, Graf N, Sakkalis V, Marias K, Wang Z, Deisboeck TS, Johnson D, et al: Dealing with diversity in computational cancer modeling. Cancer Inform 12: 115-124, 2013.

137. Loessner D, Flegg JA, Byrne HM, Clements JA and Hutmacher DW: Growth of confined cancer spheroids: A combined experimental and mathematical modelling approach. Integr Biol 5: 597-605, 2013.

138. Molina-Peña R and Álvarez MM: A simple mathematical model based on the cancer stem cell hypothesis suggests kinetic commonalities in solid tumor growth. PLoS One 7: e26233, 2012.

139. Sakkalis V, Sfakianakis S, Tzamali E, Marias K, Stamatakos G, Misichroni F, Ouzounoglou E, Kolokotroni E, Dionysiou D, Johnson D, et al: Web-based workflow planning platform supporting the design and execution of complex multiscale cancer models. IEEE J Biomed Health Inform 18: 824-831, 2014.

140. Sturrock M, Hao W, Schwartzbaum J and Rempala GA: A mathematical model of pre-diagnostic glioma growth. J Theor Biol 380: 299-308, 2015.

141. Tzamali E, Grekas G, Marias K and Sakkalis V: Exploring the competition between proliferative and invasive cancer phenotypes in a continuous spatial model. PLoS One 9: e103191, 2014.
142. He Q, Guo S, Qian Z and Chen X: Development of individualized anti-metastasis strategies by engineering nanomedicines. Chem Soc Rev 44: 6258-6286, 2015.

143. Hu C, Niestroj M, Yuan D, Chang S and Chen J: Treating cancer stem cells and cancer metastasis using glucose-coated gold nanoparticles. Int J Nanomedicine 10: 2065-2077, 2015.

144. Landesman-Milo D, Ramishetti S and Peer D: Nanomedicine as an emerging platform for metastatic lung cancer therapy. Cancer Metastasis Rev 34: 291-301, 2015.

145. Patil R, Ljubimov AV, Gangalum PR, Ding H, Portilla-Arias J, Wagner S, Inoue S, Konda B, Rekechenetskiy A, Chesnokova A, et al: MRI virtual biopsy and treatment of brain metastatic tumors with targeted nanobioconjugates: Nanoclinic in the brain. ACS Nano 9: 5594-5608, 2015.

146. Rychahou P, Shu Y, Haque F, Hu J, Guo P and Evers BM: Methods and assays for specific targeting and delivery of RNA nanoparticles to cancer metastases. Methods Mol Biol 1297: 121-135, 2015.

147. Swami A, Reagan MR, Basto P, Mishima Y, Kamaly N, Glavey S, Zhang S, Moschetta M, Seevaratnam D, Zhang Y, et al: Engineered nanomedicine for myeloma and bone microenvironment targeting. Proc Natl Acad Sci USA 111: 10287-10292, 2014.

148. Sjögren E, Westergren J, Grant I, Hanisch G, Lindfors L, Lennernäs $\mathrm{H}$, Abrahamsson $\mathrm{B}$ and Tannergren C: In silico predictions of gastrointestinal drug absorption in pharmaceutical product development: Application of the mechanistic absorption model GI-Sim. Eur J Pharm Sci 49: 679-698, 2013.

149. Jayachandran D, Ramkrishna U, Skiles J, Renbarger J and Ramkrishna D: Revitalizing personalized medicine: Respecting biomolecular complexities beyond gene expression. CPT Pharmacometrics Syst Pharmacol 3: e110, 2014.

150. Xie L, Ge X, Tan H, Xie L, Zhang Y, Hart T, Yang X and Bourne PE: Towards structural systems pharmacology to study complex diseases and personalized medicine. PLOS Comput Biol 10: e1003554, 2014.

151. Gröning R, Remmerbach S and Jansen AC: Telemedicine: Insulin pump controlled via the Global System for Mobile Communications (GSM). Int J Pharm 339: 61-65, 2007.

152. Alomari M, Mohamed FH, Basit AW and Gaisford S: Personalised dosing: Printing a dose of one's own medicine. Int J Pharm 494: 568-577, 2015.

153. Khaled SA, Burley JC, Alexander MR and Roberts CJ: Desktop $3 \mathrm{D}$ printing of controlled release pharmaceutical bilayer tablets. Int J Pharm 461: 105-111, 2014.

154. Xitian P, Hongying L, Kang W, Yulin L, Xiaolin Z and Zhiyu W: A novel remote controlled capsule for site-specific drug delivery in human GI tract. Int J Pharm 382: 160-164, 2009.

155. Farandos NM, Yetisen AK, Monteiro MJ, Lowe CR and Yun SH: Contact lens sensors in ocular diagnostics. Adv Healthc Mater 4: 792-810, 2015

156. Wening $\mathrm{K}$ and Breitkreutz J: Oral drug delivery in personalized medicine: Unmet needs and novel approaches. Int J Pharm 404: $1-9,2011$

157. Vizirianakis IS: Harnessing pharmacological knowledge for personalized medicine and pharmacotyping: Challenges and lessons learned. World J Pharmacol 3: 110-119, 2014.

158. Li M, Panagi Z, Avgoustakis K and Reineke J: Physiologically based pharmacokinetic modeling of PLGA nanoparticles with varied mPEG content. Int J Nanomedicine 7: 1345-1356, 2012.

159. Shelley ML, Wagner AJ, Hussain SM and Bleckmann C: Modeling the in vivo case with in vitro nanotoxicity data. Int $\mathbf{J}$ Toxicol 27: 359-367, 2008 .

160. Lin P, Chen JW, Chang LW, Wu JP, Redding L, Chang H, Yeh TK, Yang CS, Tsai MH, Wang HJ, et al: Computational and ultrastructural toxicology of a nanoparticle, Quantum Dot 705, in mice. Environ Sci Technol 42: 6264-6270, 2008.

161. Péry AR, Brochot C, Hoet PH, Nemmar A and Bois FY: Development of a physiologically based kinetic model for $99 m$-technetium-labelled carbon nanoparticles inhaled by humans. Inhal Toxicol 21: 1099-1107, 2009.

162. Lankveld DP, Oomen AG, Krystek P, Neigh A, Troost-de Jong A, Noorlander CW, Van Eijkeren JC, Geertsma RE and De Jong WH: The kinetics of the tissue distribution of silver nanoparticles of different sizes. Biomaterials 31: 8350-8361, 2010.

163. Mager DE, Mody V, Xu C, Forrest A, Lesniak WG, Nigavekar SS, Kariapper MT, Minc L, Khan MK and Balogh LP: Physiologically based pharmacokinetic model for composite nanodevices: Effect of charge and size on in vivo disposition. Pharm Res 29: 2534-2542, 2012. 
164. Pascal J, Ashley CE, Wang Z, Brocato TA, Butner JD, Carnes EC, Koay EJ, Brinker CJ and Cristini V: Mechanistic modeling identifies drug-uptake history as predictor of tumor drug resistance and nano-carrier-mediated response. ACS Nano 7: 11174-11182, 2013.

165. Li D, Johanson G, Emond C, Carlander U, Philbert M and Jolliet O: Physiologically based pharmacokinetic modeling of polyethylene glycol-coated polyacrylamide nanoparticles in rats. Nanotoxicology 8 (Suppl 1): S128-S137, 2014.

166. Sweeney LM, MacCalman L, Haber LT, Kuempel ED and Tran CL: Bayesian evaluation of a physiologically-based pharmacokinetic (PBPK) model of long-term kinetics of metal nanoparticles in rats. Regul Toxicol Pharmacol 73: 151-163, 2015.
167. Bachler G, Losert S, Umehara Y, von Goetz N, RodriguezLorenzo L, Petri-Fink A, Rothen-Rutishauser B and Hungerbuehler K: Translocation of gold nanoparticles across the lung epithelial tissue barrier: Combining in vitro and in silico methods to substitute in vivo experiments. Part Fibre Toxicol 12: $18,2015$.

168. Lin Z, Monteiro-Riviere NA and Riviere JE: A physiologically based pharmacokinetic model for polyethylene glycol-coated gold nanoparticles of different sizes in adult mice. Nanotoxicology 11: $1-11,2015$ 